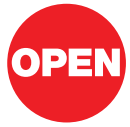

SUBJECT AREAS:

EVOLUTIONARY GENETICS

SOCIAL NEUROSCIENCE

PSYCHIATRIC DISORDERS

SOCIAL EVOLUTION

Received

29 October 2012

Accepted

6 December 2012

Published

9 January 2013

Correspondence and requests for materials should be addressed to L.J.N.B. lauren.brent@ duke.edu)

\section{Genetic origins of social networks in rhesus macaques}

\author{
Lauren J. N. Brent ${ }^{1}$, Sarah R. Heilbronner ${ }^{1,2}$, Julie E. Horvath ${ }^{3,4,5}$, Janis Gonzalez-Martinez ${ }^{6}$, \\ Angelina Ruiz-Lambides ${ }^{6}$, Athy G. Robinson ${ }^{2}$, J. H. Pate Skene ${ }^{2} \&$ Michael L. Platt ${ }^{1,2,3,7}$
}

'Duke Institute for Brain Sciences and Center for Cognitive Neuroscience, Duke University, 450 Research Drive, Durham, NC, 27708, USA, ${ }^{2}$ Department of Neurobiology, Duke University, Research Drive, Durham, NC, 27710, USA, ${ }^{3}$ Department of Evolutionary Anthropology, Duke University, Durham, NC, 27708, USA, ${ }^{4}$ Nature Research Center, Museum of Natural Sciences, Raleigh, NC, 27601, USA, ${ }^{5}$ Department of Biology, North Carolina Central University, Durham, NC, 27707, USA, ${ }^{6}$ Caribbean Primate Research Center, University of Puerto Rico, Medical Sciences Campus, PO Box 906, Punta Santiago, PR, 00741, USA,

7 Department of Psychology \& Neuroscience, Duke University, 417 Chapel Drive, Durham, NC, 27708, USA.

Sociality is believed to have evolved as a strategy for animals to cope with their environments. Yet the genetic basis of sociality remains unclear. Here we provide evidence that social network tendencies are heritable in a gregarious primate. The tendency for rhesus macaques, Macaca mulatta, to be tied affiliatively to others via connections mediated by their social partners - analogous to friends of friends in people - demonstrated additive genetic variance. Affiliative tendencies were predicted by genetic variation at two loci involved in serotonergic signalling, although this result did not withstand correction for multiple tests. Aggressive tendencies were also heritable and were related to reproductive output, a fitness proxy. Our findings suggest that, like humans, the skills and temperaments that shape the formation of multi-agent relationships have a genetic basis in nonhuman primates, and, as such, begin to fill the gaps in our understanding of the genetic basis of sociality.

ociality - the tendency to interact with others in an affiliative and agonistic manner - is believed to have evolved as a strategy for animals to cope with challenges in their environment ${ }^{1,2}$. Endorsing this hypothesis, social behaviours, especially affiliative ones, are positively associated with reproductive success and longevity in a number of species (e.g. $\left.{ }^{3-5}\right)$. However, in order for sociality to evolve it must have a heritable, genetic, basis on which selection may act ${ }^{5,6}$. Yet, whether sociality demonstrates additive genetic variance, and which genes contribute to such variation, remains poorly understood.

Pairs of group-living animals do not interact in a social vacuum but within an interconnected network of social relationships ${ }^{7,8}$. Studying the genetic basis of sociality requires quantitative measures that accurately reflect the complexity of this trait. Social network analysis is an increasingly popular tool for studying complex social systems as it quantifies the extent to which an individual is connected to others within the topology of the network as a whole ${ }^{8}$. Network metrics of sociality include measures that reflect direct connections - A interacts with B - as well as indirect connections - A and C interact independently with B, thus A and C are indirectly connected via B. Indirect connections, often thought of as "friend of a friend" relationships in humans, can influence factors such as dominance rank $^{9}$ and well-being ${ }^{10,11}$, and may be especially important to animals that form highly differentiated relationships and recognize bonds between pairs of third parties ${ }^{7}$.

To our knowledge, only two previous quantitative genetic studies have examined the heritable basis of social networks. In a study of human friendship, the number of times a subject was named as the friend of others demonstrated additive genetic variance ("indegree" $h^{2}=0.46$ ), as did two measures of indirect connectedness, the proportion of an individual's friends who are friends with one another ("clustering coefficient" $h^{2}=0.47$ ), and the extent to which an individual connects pairs of individuals who are not friends with each other ("betweenness" $\left.h^{2}=0.29\right)^{12}$. In marmots (Marmota flaviventris), indegree was heritable in both affiliative and agonistic networks (in marmots, indegree represents the number of partners from which a subject receives interactions: affiliation indegree $h^{2}=0.11$; agonistic indegree $h^{2}=0.11$ ), as was a second measure of direct agonistic connections ("attractiveness" $h^{2}=0.18$ ), but none of the evaluated measures of indirect connectedness demonstrated additive genetic variance ${ }^{5}$. This difference in the heritability of indirect connectedness between humans and marmots may reflect differences in the social systems of these species, including differences in the importance of third party relationships. 
Here we explore the heritable basis of sociality in a highly gregarious species of nonhuman primate. In a free-ranging population of rhesus macaques (Macaca mulatta), we examined genetic variation associated with both affiliative and agonistic interactions; no study has explored the latter in human or nonhuman primates. We also set out to determine whether, as in humans, the tendency to form indirect connections has a genetic basis in rhesus macaques. Prior studies have shown that rhesus macaques exhibit high levels of affiliation and aggression ${ }^{13}$, occupy differentiated positions within their social networks ${ }^{10,14}$, recognise relationships between third parties ${ }^{15}$, and are sensitive to the perspectives of others ${ }^{16}$.

For sociality to evolve, natural selection acts on genetic variation and its component social behaviours via the influence of those behaviours on fitness. While previous studies have shown that affiliative behaviours predict reproductive success in some primates ${ }^{4,17-19}$, much less is known about the relationship between agonism and fitness. Thus, we also explored the association between fitness and both affiliative and agonistic social interactions. Finally, recent studies have linked genes in the serotonin pathway to variation in social behaviour in humans and captive primates ${ }^{20-24}$. Nevertheless, the association between serotonergic polymorphisms and naturally occurring social behaviours is less clear. We further aimed to investigate whether genes in this key neuromodulatory system contribute to social variation in our free-ranging population.

To address these questions, we examined networks derived from affiliative (grooming and spatial proximity) and aggressive interactions separately based on two years of data (Fig. 1). For each of these networks, for each year, we calculated an individual-based measure of direct connectedness, strength. Strength is the "weighted" version of degree ${ }^{8}$ and represents the total frequency at which an individual engages in a given interaction with all partners. We calculated both instrength and outstrength - the frequencies at which an individual receives and gives interactions, respectively - for interactions that can be assigned direction (grooming, aggression). For example, grooming instrength is the rate as which an individual receives grooming
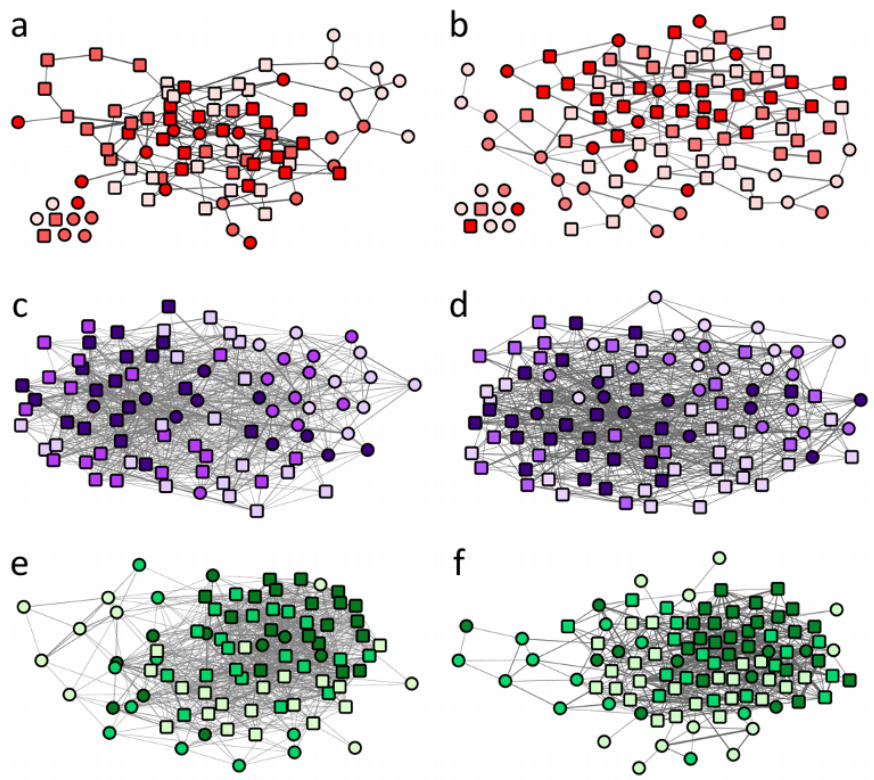

Figure 1 Social networks of adult rhesus macaques. Networks represent grooming $(\mathrm{a}, \mathrm{b})$, aggression $(\mathrm{c}, \mathrm{d})$, and spatial proximity $(\mathrm{e}, \mathrm{f})$ interactions observed in 2010 (a, c, e), and 2011 (b, d, f). Nodes represent individual animals $(n=87$ in 2010, $n=98$ in 2011). Females are squares, males circles. Node colour represents dominance rank, increasing from low (pale colours), to mid (medium colours), to high rank (dark colours). Lines represent the presence of an interaction between a pair of individuals with line thickness increasing with the frequency of interaction. from others, while aggression outstrength is the rate at which an individual gives aggression to others. For proximity, which has no direction, strength is the total amount of time a subject spends in proximity to others. We also calculated two measures of indirect connectedness. Betweenness represents the total number of shortest paths in a network graph that pass through a given individual to connect others ${ }^{25}$. Individuals with high betweenness connect pairs of individuals who are not otherwise connected to each other (e.g. they are grooming partners with pairs of individuals who are not grooming partners with each other). Finally, we calculated eigenvector, a measure of the extent to which an individual's social partners are themselves connected ${ }^{25}$. Individuals with high eigenvector interact with others at a high frequency and have partners who interact with others at a high frequency as well (e.g. they spend a lot of time grooming, and have partners who themselves spend a lot of time grooming).

We hypothesized that sociality is heritable and predicted that both direct and indirect measures of sociality would demonstrate additive genetic variance. In addition, we predicted that outwardly directed measures of sociality (outstrength) would demonstrate greater levels of additive genetic variance than inwardly directed measures (instrength) since the former represent interactions over which an individual has a greater amount of control (c.f. ${ }^{5}$ ). We also quantified the magnitude of selection for all traits based on their association with individual reproductive output, and explored genotypic and phenotypic collinearity between traits to allow more complete interpretation of our results. Finally, we examined the possible association between measures of sociality demonstrating heritable variation and variation in genes involved in serotonergic signalling. We focused specifically on a variable insertion in the gene encoding tryptophan hydroxylase (TPH2), the rate limiting enzyme in serotonin synthesis, and the 5-HTTLPR polymorphism within the promoter region of the serotonin transporter gene (SLC6A4).

\section{Results}

Heritability of sociality. We found additive genetic variance for some measures of sociality, in particular those representing indirect connections between individuals. These results were found while controlling for environmental and other factors, such as age, sex, and dominance rank, as well as social "household" effects, which represented matriline (for females) and natal group (for males). Grooming betweenness, an index of affiliative social positioning, demonstrated significant additive genetic variance $\left(h^{2}=0.84, P=\right.$ $0.025)$, while eigenvector for grooming and spatial proximity demonstrated trends toward additive genetic variance $\left(h^{2}=0.36\right.$, $P=0.073$ and $h^{2}=0.33, P=0.060$, respectively) (Table 1 ), consistent with a genetic basis for the tendency to interact frequently in an affiliative manner with partners who themselves interact affiliatively at high rates with others. Finally, aggression outstrength, a measure of the frequency at which individuals direct aggression toward others, demonstrated significant additive genetic variance $\left(h^{2}=0.66, P=0.020\right)$ (Table 1$)$. Following predictions, but in contrast to previous findings in humans and marmots, we found outward directed measures of sociality had greater estimates of heritability (grooming outstrength $h^{2}=0.31$, aggression outstrength $h^{2}=0.66$ ) compared to inward directed measures (grooming instrength $h^{2}=0.00$, aggression instrength $h^{2}=0.00$ ), but only aggression outstrength was statistically significant (Table 1 ).

Relationship between sociality and reproductive success. Measures of sociality with additive genetic variance greater than zero have the potential to influence evolution by natural selection. Of these measures, we found that proximity eigenvector had a significant selection differential (Table 1): as proximity eigenvector increased, so too did the number of offspring produced by an individual (Fig. 2a). The relationships between reproductive output and many 


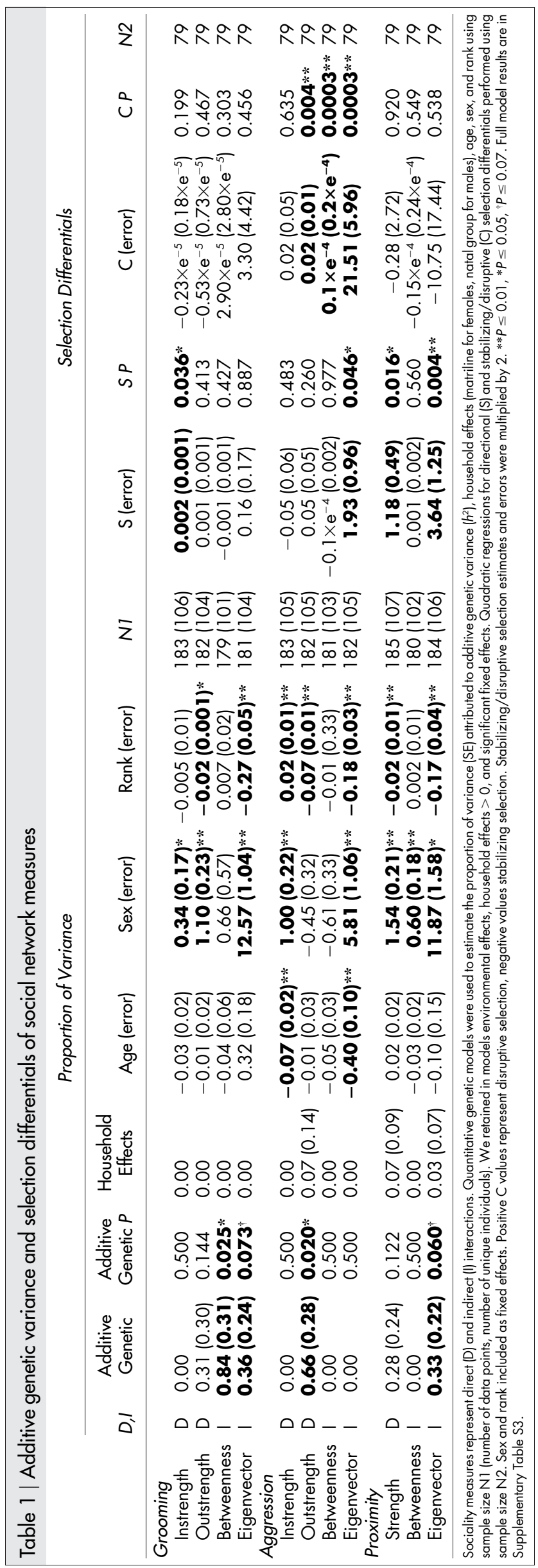



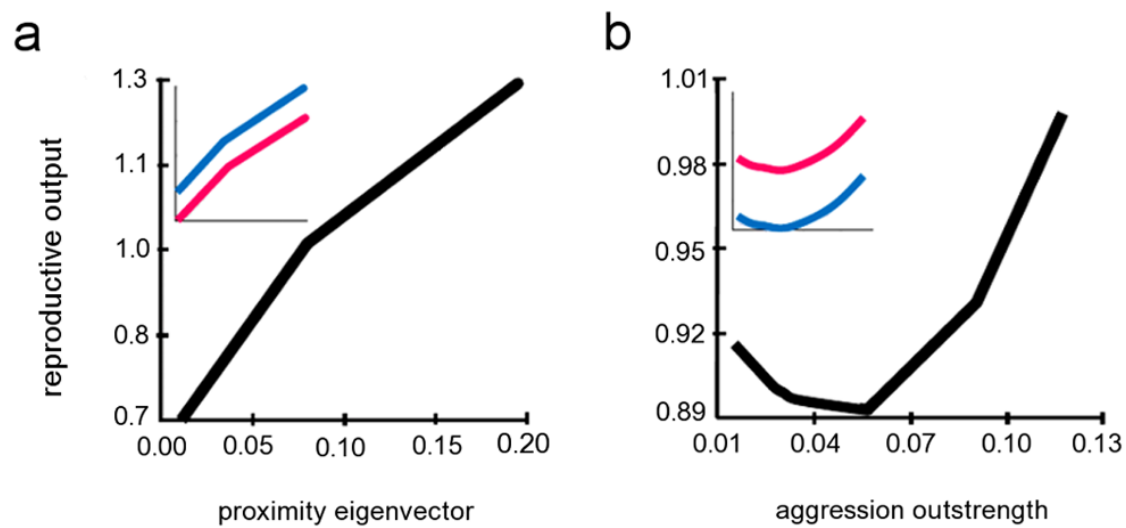

Figure $2 \mid$ Predictive plots for the relationship between sociality and relative reproductive output. Plots control for sex and rank. (a) Proximity eigenvector - the tendency of an individual to spend time in proximity with individuals who themselves spend time in proximity to others - increased significantly along with reproductive output. (b) The relationship between aggression outstrength and reproductive output was statistically significant and consistent with disruptive selection. Individuals with the greatest reproductive output directed both the lowest and highest amounts of aggression to others. Relationships for males (blue, $n=29$ ) and females (pink, $n=58$ ) shown in inset graphs. Plots generated in $\mathrm{MLwiN}^{58}$.

measures of aggression were statistically significant and consistent with disruptive selection; individuals in the tail ends of the distribution of aggressive tendencies produced greater numbers of offspring relative to other members of their age cohort and sex (Table 1). For example, individuals who gave the lowest amounts of aggression to others (low aggression outstrength), as well as those who gave the greatest amounts of aggression (high aggression outstrength) produced the greatest relative number of offspring (Fig. 2b). Because the size of some of these effects were small (e.g. aggression outstrength estimate $+/-\mathrm{SE}=0.02+/-0.01$, Table 1 ), they should be interpreted with caution.

Phenotypic and genotypic co-variance of sociality measures. Many of the traits we examined co-varied at the phenotypic level, such that individuals with a high score for one metric tended to have high scores for the other metrics (these results are presented in Supplementary Table S1). However, none of the traits that demonstrated additive genetic variance were genetically correlated (Supplementary Table S2). Similar genes do not therefore appear to contribute to the expression of variation in grooming eigenvector, grooming betweenness, proximity eigenvector, and aggression outstrength in this population.

Consistency in sociality measures over time. Individuals did not differ in their social network positions from one year to the next (Supplementary Fig. S4), apart from proximity strength, which showed significant differences $(P=0.001)$. The amount of time individuals spend in proximity to others does not, therefore, exhibit within-individual consistency, and although not statistically significant in genetic analyses, results for this measure should be interpreted carefully.

Sociality and serotonergic genes. Of the traits whose phenotypic variation was explained by additive genetic variance (either significantly, or with a strong trend toward significance), we found one to be associated with serotonergic gene profiles. Grooming eigenvector was significantly related to the interaction between the two polymorphisms examined ( $P=0.037$, Table 2$)$, whilst controlling for factors such as dominance rank and including information on relatedness between subjects (Fig. 3). Although the significance of this result in the multivariate model did not withstand correction for multiple tests (Bonferroni $\alpha=0.012$ ), post-hoc analysis of this relationship showed that individuals that carried both the 5-HTTLPR short (S) allele and the TPH2 long (L) allele had significantly lower grooming eigenvector levels than individuals with other combinations of variants at these two loci (Fig. 3f, i) (twotailed t-tests for 2010 data: $t=2.63, P=0.011$, and 2011 data: $t=$ $4.18, P=0.00007)$. These alleles were reported as minor alleles based on their frequency in this (see Fig $3 e$ and Supplementary Fig. S1), as well as other, populations. Thus, individuals carrying the minor alleles for both of these genes were less well-connected in the grooming network compared to individuals with other serotonergic gene profiles (Fig. $3 \mathrm{~h}, \mathrm{k}$ ). In addition, although broadly distributed, the $95 \%$ confidence intervals of post-hoc analysis values did not overlap the value which would indicate no significant effect (i.e. zero) $\left(C I_{2010}=1.23-8.99, C_{2011}=2.40-6.75\right)$ further supporting an

Table 2 | Relationship between sociality and genes in the serotonin pathway

\begin{tabular}{|c|c|c|c|c|c|c|c|}
\hline & \multicolumn{2}{|c|}{ 5-HTTLPR } & \multicolumn{2}{|c|}{$\mathrm{TPH} 2$} & \multicolumn{2}{|c|}{ 5-HTTLPR *TPH2 } & $N$ \\
\hline \multicolumn{8}{|l|}{ Grooming } \\
\hline Eigenvector & $0.32(1.03)$ & 0.678 & $1.55(1.02)$ & 0.133 & $-3.46(1.67)$ & $0.037 *$ & $181(104)$ \\
\hline \multicolumn{8}{|l|}{ Aggression } \\
\hline Outstrength & $-0.43(0.38)$ & 0.150 & $-0.78(0.40)$ & 0.065 & $0.57(0.57)$ & 0.283 & $182(105)$ \\
\hline \multicolumn{8}{|l|}{ Spatial Proximity } \\
\hline
\end{tabular}




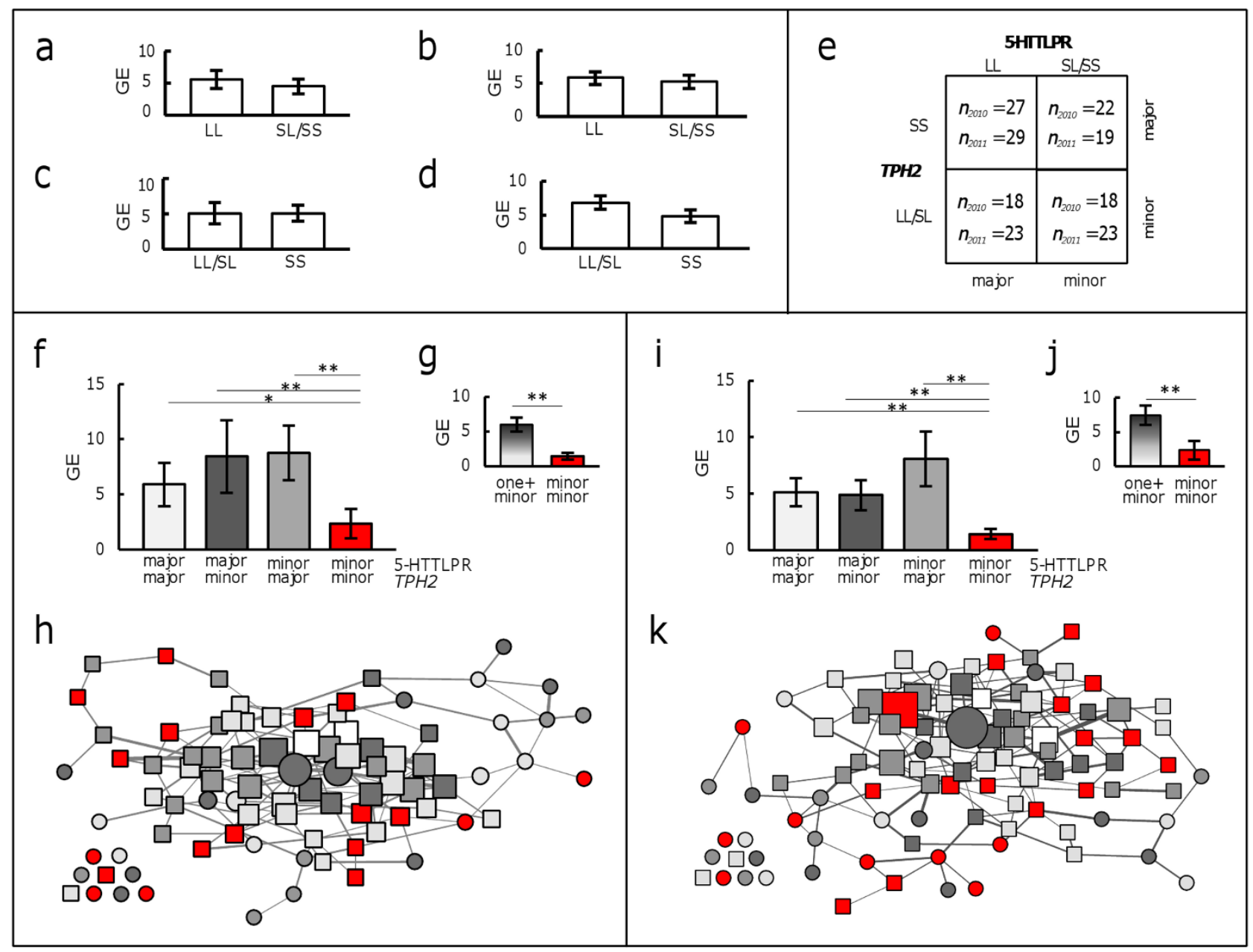

Figure $3 \mid$ Association between grooming and serotonergic gene profiles. Individuals were grouped according to presence of minor (less frequent) alleles, $\mathrm{S}$ for 5-HTTLPR, L for TPH2. We found no significant association between grooming eigenvector (GE) and the 5-HTTLPR (a, b) or TPH2 (c, d) polymorphisms as main effects in either year of study $(2010: \mathrm{a}, \mathrm{c} ; 2011 \mathrm{l}$ b,d). (bar graphs $=$ mean \pm SE). The interaction between these variables was a significant predictor of GE in quantitative genetic analyses $(P=0.037)$. Based on this interaction $(\mathrm{e})$, individuals had major alleles at both loci $\left(n_{2010}=27\right.$; $\left.n_{2011}=29\right)$, the major allele at one loci and minor allele at the other $\left(n_{2010}=18\right.$ and $22 ; n_{2011}=23$ and 19$)$, or minor alleles at both loci $\left(n_{2010}=18 ; n_{2011}=\right.$ 23). Individuals with minor alleles at both loci had significantly lower GE scores than individuals with at least one major allele in both 2010 (f) and 2011 (i). GE for individuals with at least one major allele did not differ significantly and these data were collapsed (g, j) (see also Supplementary Fig. S3 for sample sizes and mean GE using raw data for all possible gene profiles). Two-tailed t-tests of collapsed data were statistically significant (2010: $t=2.63$, $P=0.011 ; 2011: t=4.18, P=0.00007)$. Sociograms of the grooming networks in $2010(\mathrm{~h})$ and $2011(\mathrm{k})$. Squares are females, circles males. Lines represent the presence of a grooming interaction between dyads with line thickness increasing with frequency of interaction. Individuals with the highest GE are represented by largest node sizes and central graph positions. Based on their serotonergic gene profiles, rhesus macaques with minor alleles at both loci (red nodes) had the lowest GE. These individuals have small node sizes and are toward the graph's periphery, sometimes with no grooming partners (nodes in bottom left). Two females homozygous XL for 5-HTTLPR (white nodes) were excluded from analyses. ${ }^{* *} P \leq 0.01, * P \leq 0.05$.

effect of serotonin gene profile on grooming eigenvector ${ }^{26}$. No other significant relationships were found between other traits and these loci as main effects, or as interaction terms (Table 2).

\section{Discussion}

Sociality is assumed to be adaptive, shaped by natural selection acting on heritable variation ${ }^{1,2}$, but its genetic basis remains poorly understood. Here, we report that sociality demonstrates additive genetic variance in a free-ranging population of primates, and is thus heritable. In particular, the tendency to direct aggression toward others, as well as the tendency to form indirect affiliative relationships were both found to have a heritable basis. Indirect connections in humans friend of a friend relationships - have been reported to have a similar underlying genetic structure ${ }^{12}$, but only direct measures of sociality were found to have a heritable basis in marmots ${ }^{5}$.
Recognising the relationships of others is a crucial component of social intelligence that has been well-documented in primates ${ }^{27}$. Nevertheless, how animals use this information and whether or not it has been shaped by natural selection remains largely unknown ${ }^{27}$. Taken with previous findings in humans and marmots, our results suggest that social temperaments and skills that shape indirect connections may have a genetic basis in some highly gregarious taxa such as the Primates, but perhaps not in other animals, although studies in a broader range of species are required to confirm this idea. Sociality appears to be under selective pressure as well: we found a positive association between indirect measures of sociality and reproductive output. Together, these results endorse the idea that the capacity to form social relationships mediated by other individuals has been shaped by natural selection and may play a crucial role in the evolution of primate societies. 
The association between affiliative social behaviours and fitness confirms previous findings in primates-individuals with the greatest indices of sociality (comprised of both grooming and spatial proximity interactions) have been shown to have higher infant survival rates and greater longevity (female baboons, Papio spp. $)^{4,18,19}$, and to produce the greatest number of offspring (male Assamese macaques, $M$. assamensis $)^{17}$. Much less is known, however, about the association between agonistic interactions and fitness. Our study found that aggressive tendencies were significantly related to reproductive output in a way consistent with disruptive selection. Disruptive selection acts to push the distribution of a trait towards its extremes ${ }^{28}$. In this case, there was an association between reproductive output and both low and high levels of aggression. Thus, there may be two alternative routes to success in rhesus macaques-an aggressive route and a passive route. However, it should be noted that heritability estimates were only significant for one measure of aggression, the extent to which individuals direct aggression toward others. A lack of additive genetic variance may suggest the other measures of aggressiveness do not have a genetic basis and therefore no evolutionary potential, although studies of different populations in a variety of contexts are required before such a statement be adopted with confidence. Alternatively, the association with fitness we found may indicate that selection has acted to reduce genetic variation in aggression ${ }^{29,30}$. If true, this could signify that aggressive strategies are vital to the success of male and female rhesus macaques. However, small selection differentials could also indicate that the size of the effect of aggression on reproductive output is below that which might be expected to influence selection.

In our study, one measure of sociality was associated with serotonergic gene profiles based on the 5-HTTLPR and TPH2 polymorphisms. Grooming eigenvector, the tendency of individuals to spend a lot of time grooming partners who themselves spend a lot of time grooming their own partners, was significantly related to the interaction between the two polymorphisms examined. Although the significance of this result did not withstand correction for multiple hypothesis testing and should, therefore, be considered preliminary until replicated, it is nevertheless consistent with previous studies linking these loci to variation in social behaviour ${ }^{31}$. Serotonin is involved in the regulation of mood, memory and learning ${ }^{32}$. Previous studies in humans and captive rhesus macaques have linked the $S$ allele of the 5-HTTLPR polymorphism to elevated levels of anxiety, elevated response to social threats, and to a tendency to avoid risks ${ }^{2021,23,33}$, while polymorphisms in the gene coding for TPH2 have been associated with human pathologies such as autism spectrum disorder and depression ${ }^{22,34,35}$. In addition, an additive effect of these two genes has been reported to underlie differences in human brain activity in response to social stimuli, with the greatest activation occurring in individuals with gene profiles predicted to result in the greatest recovery of synaptic serotonin ${ }^{36}$.

By altering expression of genes involved in serotonin synthesis and reuptake, respectively, different combinations of TPH2 and 5HTTLPR alleles are expected to influence the intensity and duration of signalling at serotonergic synapses in the brain. The serotonin transporter mediates reuptake of serotonin at synapses, which serves both to terminate serotonergic signalling and to replenish serotonin in presynaptic terminals. Carriers of the minor 5-HTTLPR allele (S) produce lower levels of serotonin transporter, which results in prolonged serotonergic signalling in the short term but depletes presynaptic stores over time ${ }^{37}$. Increased TPH2 production, observed in carriers of the minor allele of the TPH2 repeat polymorphism (L), support higher rates of serotonin production and thus maintain higher levels of presynaptic serotonin ${ }^{38}$, although increased TPH2 production has also been associated with decreased recovery of serotonin to the presynaptic terminal over time ${ }^{24}$. In addition, variation in these genes has been correlated with altered development of several brain regions $s^{37,39}$. Although tentative, the association between serotonergic gene profiles and grooming behaviour in the current study appears to be consistent with a model in which the interaction between the 5-HTTLPR and TPH2 polymorphisms results in suboptimal levels of serotonergic signaling and/or with poorly developed neural circuits, and which subsequently results in rhesus macaques that are poorly-connected in an affiliative manner.

In combination with previous findings, our results may also be used to address tentatively why the minor alleles for these genes persist in the population. Social integration was reduced only for rhesus macaques carrying the minor alleles of both TPH 2 and SLC6A4 in this study; individuals carrying the minor allele for either gene alone were no less socially connected than homozygotes for both major alleles. In addition to no effect on social integration, rhesus macaques with the minor allele for either gene alone may fare better under highly uncertain social and environmental conditions, capitalizing on a tendency to be more vigilant to threats and less likely to adopt risky strategies ${ }^{23}$. Indeed, the 5-HTTLPR polymorphism does not predict reproductive output for males in the Cayo Santiago population ${ }^{40}$, a finding that has been related to the association between this genotype and variation in the age males disperse from their natal groups ${ }^{41}$. Together, these findings suggest variation at this locus is either maintained by, or is neutral to, natural selection. Future studies of the association between genetic variation and behaviour, particularly those focusing on naturally occurring behaviours, may reveal a clearer understanding of the costs and benefits of different serotonergic genotypes.

Previous quantitative genetic studies have uncovered specific gene by environmental interactions ${ }^{33,42}$. Individual rhesus macaques may be more affiliative because they have certain gene profiles, or because they have been subject to particular experiences, which promote different behavioural tendencies. Epigenetic factors, such as DNA methylation, can also vary with environment ${ }^{43}$, and may also contribute. On a broad-scale, environment was consistent in our study (i.e. subjects were in the same social group during the same period of time, while food provisioning minimizes variation in caloric intake, and factors such as natal group were accounted for statistically), and we accounted for the impact of social factors (e.g. matriline, natal group) on phenotypic variance whenever possible. Nevertheless, environmental, epigenetic, and early-life factors that could not be taken into account using the current dataset are likely to play an important role in the expression of social behaviours in this population. Moreover, other putative polymorphisms exist in the serotonin pathway in this species ${ }^{44}$. Assessment of their distributions and associations to sociality will illuminate the relationship between these traits and their underlying genetic structure.

Sociality is an enigmatic trait found in a number of group-living animals including humans and many other species of primate. Our findings echo previous work showing a quantitative link between variation in sociality and fitness. This study also begins to fill the genetic gaps in our understanding of the evolution of sociality, particularly the indirect, multi-agent interactions that characterize the social behaviour of humans and nonhuman primates alike.

\section{Methods}

Study subjects and behavioural data collection. Subjects were adult members of a single group of free-ranging rhesus macaques (group " $F$ ", $n=107$ ) living on Cayo Santiago Island, Puerto Rico. This population was established in 1938 with a single founding population from India ${ }^{45}$. Subjects were individually recognized and habituated to observers. Animals are provisioned daily with commercial feed, and browse on natural vegetation with water supplied ad libitum. Population control, involving the removal of mostly juveniles, is undertaken once yearly. Animals are not handled outside the removal period and there is no regular medical intervention.

Rhesus macaques (Macaca mulatta) are Old World monkeys with a well-characterized social system including high levels of affiliation and despotism, characterized by intense unidirectional aggression from dominants to subordinates ${ }^{13}$. Females are philopatric, while males usually leave natal groups at puberty. Male dominance rank is established by physical combat and is transient. Females form stable linear dominance hierarchies, with females securing ranks immediately below their mothers ${ }^{46}$ 
The female hierarchy is further structured into matrilines, made up of closely related females that are close in rank.

We collected behavioural data between May-Dec 2010, and April-Dec 2011 using 10-minute continuous focal animal samples on adults subjects, six-25 years of age, mean $(\mathrm{SD})=12.34$ (4.48). Group F contained 29 adult males and 58 adult females in $2010(n=87)$, and 32 adult males and 66 adult females in $2011(n=98)$. The increase in female numbers was due to maturation of juveniles to adults. Females belonged to three matrilines, and there were no major shifts in rank in the male hierarchy. Six mid to low ranking individuals died and were excluded from analyses, as were five midranking males who emigrated from the group at the beginning of 2011.

We collected 843.7 total hrs of focal animal data with means (SD) of 4.07 (0.39) and $5.02(0.11)$ hrs per subject in 2010 and 2011, respectively. We controlled for time of day with data collection evenly spread throughout the day between and within subjects. We recorded the occurrence of all social behaviours, including the identity of the focal animal's social partner. Aggression included contact and non-contact aggression. We recorded the identities of all adults in proximity $(\leq 2 \mathrm{~m})$ to the focal animal at five-minute intervals for a total of three data points/focal animal sample. We determined dominance rank from the direction and outcome of submissive interactions.

Social network measures. We constructed social networks independently for each year of data collection using UCINET ${ }^{47}$. We normalised network metrics by expressing an individual's score as a percentage relative to the maximum possible score in each year. We generated confidence intervals for network metrics from 1,000 bootstrapped replicates of association matrices ${ }^{48}$ using the "tnet" package in $\mathrm{R}^{49}$. Many individuals exhibited considerable differences in network position from each other (Supplementary Fig. S2).

In order to determine whether individuals were consistent in their network positions from one year to the next, we performed pair-wise comparisons of individual network positions for subjects that were present in both years $(n=78)$ using the means of our bootstrapped data via permutation-based t-tests using PSAM software $(10,000 \text { permutations })^{50}$. We controlled for the impact of differences in rank between years by dividing an individual's network position in a given year by their rank in that year.

To confirm our quantitative genetic results, we re-ran analyses using mean network measures generated from random networks $(n=1,000)$ with the same number of individuals and probability of association as observed networks. We found no evidence of additive genetic variance for any sociality measure based on random networks (Supplementary Table S4).

Pedigree. Parentage assignment was based on 29 microsatellite markers (see Supplementary Methods). Among subjects, there were 12 parent-offspring pairs but no full siblings - mean $r(\mathrm{SD})=0.02(0.05)$ (Supplementary Figure S3). There is little evidence for high rates of inbreeding on Cayo Santiago ${ }^{51}$.

Quantitative genetic analyses. For all quantitative genetic analyses, we used a variance components model in the program Sequential Oligonucleotide Linkage Analysis Routines $\left(\mathrm{SOLAR}^{52}\right)$. This model accommodates covariate effects and is well-suited to extended pedigrees such as ours. We tested the significance of heritability estimates by comparing a model that assumes no genetic effect on phenotypic variance to one that assumes an effect of genetics. We evaluated each social network measure using a univariate model that included individual-based environmental effects as well as "household" effects ${ }^{52,53}$. Household effects $\left(c^{2}\right)$ were matriline for females and natal group for males, the dispersing sex. We included age, dominance rank (on a continuous scale) and sex as fixed effects. Prior to variance component analyses, we blanked outlying values for each network measure ${ }^{53}$. Transformation of data to a normal distribution was often not possible due to positive skew. We therefore used a multivariate $t$ distribution, rendering this analysis robust to non-normally distributed data ${ }^{52,54}$.

We calculated phenotypic collinearity between all pairs of network measures using Spearman rank correlation $s^{30}$ in R (results are in Supplementary Table S1). For network measures with heritability estimates $>0$, we calculated genetic correlations using bivariate variance components analyse ${ }^{55}$, including all fixed and random effects from heritability analyses (results are in Supplementary Table S2).

We assessed the fitness consequences of social network metrics using adjusted number of offspring produced - extracted from the pedigree - as a measure of reproductive output. We used social network metrics based on 2010 data only as parentage assignment was not completed beyond this year. Females sired an average (SD) of 7.21 (4.07) offspring, males 9.82 (9.18). Variance is greater in males, consistent with male reproductive skew in this species ${ }^{56}$. We adjusted values for each subject relative the mean number of offspring for other individuals of that sex and age cohort, allowing us to account for increases in the number of offspring as a function of age, as well as to cope with the effect of heritability by reducing the number of individuals whose reproductive output was adjusted relative to that of their close relatives (e.g. parent-offspring pairs) ${ }^{57}$. For both sexes, values represent number of offspring to survive to one year as DNA is not routinely collected for infants who die prior to one year, and parentage cannot be assigned using genetics. While maternity is known through behaviour even if the infant dies prior to DNA collection, the number of infants who did not survive to one year was minimal (0.002\%).

Female kin-biased interactions did not appear to substantially impact the relationship between sociality and reproductive output in this study as females with adult daughters present did not have greater reproductive output, nor were they more socially central, compared to females without adult daughters (see Supplementary Methods).

We used univariate quadratic regressions to estimate selection differentials. We estimated linear selection ( $S$ ) as the linear covariance between each network metric and reproductive output, and nonlinear selection $(\mathrm{C})$ as the covariance between reproductive output and the orthogonal quadratic estimate of the metric ${ }^{28}$. We doubled quadratic regression coefficients and standard errors in order to correctly estimate selection gradients $s^{57}$. Negative quadratic estimates represent evidence of stabilizing selection, positive estimates represent disruptive selection. We included sex and rank as fixed effects in all cases. We fit selection differential models using MCMC estimations in MLwiN 2.2358.

DNA sample collection. We obtained whole blood from live-trapped animals during periods in which behavioural data were not collected (Jan-Mar 2009, 2010, and 2011). Animals were netted following entry to an enclosed area and anesthetized using an injection of ketamine (10 mg/kg body weight). We collected blood via venous puncture and isolated DNA in the field using a QIAGEN kit (cat. \#51106).

Serotonin polymorphisms. We assessed polymorphisms using PCRs with previously described primers. The 5-HTTLPR polymorphism of the serotonin transporter gene (SLC6A4) and the TPH2 repeat polymorphism (159 bp insertion in the $3^{\prime}$ UTR) are both characterized by long $(\mathrm{L})$ and short $(\mathrm{S})$ numbers of repeat $\mathrm{s}^{37,38}$. 5-HTTLPR also has a rare extra-long $(\mathrm{XL})$ allele ${ }^{59}$. Allelic distributions in our study $(n=107)$ were: 5-HTTLPR: $\mathrm{LL}=50.5 \%, \mathrm{SL}=39.3 \%, \mathrm{SS}=8.4 \%, \mathrm{XL}=1.9 \%$; $\mathrm{TPH} 2 \mathrm{LL}=4.7 \%, \mathrm{SL}=$ $43.0 \%$, SS $=52.3 \%$, which are similar to those of previously published finding ${ }^{38,40}$ and do not deviate from Hardy-Weinberg equilibrium (5-HTTLPR: $P=0.785$; TPH2: $P=0.276$ ). These loci demonstrated little evidence of linkage disequilibrium, with $\mathrm{D}$ at only $6 \%$ of its maximal possible value ${ }^{60}$. In order to evaluate the association between these polymorphisms and sociality, we included these loci and the interaction between them as fixed factors in quantitative genetic analyses along with random and fixed effects from heritability analyses, as well as pedigree information, thus appropriately accounting for relatedness between subjects ${ }^{55}$. We grouped individuals that carried at least one copy of the minor allele for each locus as is the standard for studies with few homozygotes ${ }^{36,53}$. We defined minor alleles based on their frequency, with the minor alleles being the $\mathrm{S} 5$-HTTLPR allele, and the $\mathrm{L} T P H 2$ allele (Fig. 3a, b). We excluded the 5-HTTLPR XL allele due to rarity.

This study was approved by the IACUC of the University of Puerto Rico, protocol \# A6850108.

1. Wilson, E. O. Sociobiology: The New Synthesis (The Belknap Press of Harvard University Press, 1975).

2. Cheney, D., Seyfarth, R. \& Smuts, B. Social relationships and social cognition in nonhuman primates. Science 234, 1361-1366 (1986).

3. Cameron, E. Z., Setsaas, T. H. \& Linklater, W. L. Social bonds between unrelated females increase reproductive success in feral horses. Proc. Natl. Acad. Sci. 106, 13850-13853 (2009).

4. Silk, J. B., Alberts, S. C. \& Altmann, J. Social bonds of female baboons enhance infant survival. Science 302, 1231-1234 (2003).

5. Lea, A. J., Blumstein, D. T., Wey, T. W. \& Martin, J. G. A. Heritable victimization and the benefits of agonistic relationships. Proc. Natl. Acad. Sci. 107, 21587-21592 (2010).

6. Charmantier, A., Buoro, M., Gimenez, O. \& Weimerskirch, H. Heritability of short-scale natal dispersal in a large-scale foraging bird, the wandering albatross. J. Evol. Biol. 24, 1487-1496 (2011).

7. Brent, L. J. N., Lehmann, J. \& Ramos-Fernández, G. Social network analysis in the study of nonhuman primates: A historical perspective. Am. J. Primatol. 73, 720-730 (2011)

8. Croft, D. P., James, R. \& Krause, J. Exploring Animal Social Networks (Princeton University Press, 2008).

9. McDonald, D. B. Predicting fate from early connectivity in a social network. Proc. Natl. Acad. Sci. 104, 10910-10914 (2007).

10. Brent, L. J. N., Semple, S., Dubuc, C., Heistermann, M. \& MacLarnon, A. Social capital and physiological stress levels in free-ranging adult female rhesus macaques. Physiol. Behav. 102, 76-83 (2011).

11. Fowler, J. H. \& Christakis, N. A. Dynamic spread of happiness in a large social network: longitudinal analysis over 20 years in the Framingham Heart Study. Br. Med. J. 337, a2338 (2008).

12. Fowler, J. H., Dawes, C. T. \& Christakis, N. A. Model of genetic variation in human social networks. Proc. Natl. Acad. Sci. 106, 1720-1724 (2009).

13. Thierry, B. Social Epigenisis. In Macaque Societies: A Model for the Study of Social Organization (eds Thierry, B., Singh, M. \& Kaumanns, W.) Ch. 12, 267-290 (Cambridge University Press, 2004).

14. Sade, D. S. Sociometrics of Macaca mulatta I: Linkages and cliques in grooming matrices. Folia Primatol. 18, 196-223 (1972).

15. Gouzoules, S., Gouzoules, H. \& Marler, P. Rhesus monkey (Macaca mulatta) screams: Representational signaling in the recruitment of agonistic aid. Anim. Behav. 32, 182-193 (1984).

16. Flombaum, J. I. \& Santos, L. R. Rhesus monkeys attribute perceptions to others. Curr. Biol. 15, 447-452 (2005).

17. Schülke, O., Bhagavatula, J., Vigilant, L. \& Ostner, J. Social bonds enhance reproductive success in male macaques. Curr. Biol. 20, 1-4 (2010). 
18. Silk, J. B. et al. The benefits of social capital: close social bonds among female baboons enhance offspring survival. Proc. Roy. Soc. B. 276, 3099-3104 (2009).

19. Silk, J. B. et al. Strong and consistent social bonds enhance the longevity of female baboons. Curr. Biol. 20, 1359-1361 (2010).

20. Hariri, A. R. et al. Serotonin transporter genetic variation and the response of the human amygdala. Science 297, 400-403 (2002).

21. Lesch, K. P. et al. Association of anxiety-related traits with a polymorphism in the serotonin transporter gene regulatory region. Science 274, 1527-1531 (1996).

22. Chen, G. L. et al. The effect of rearing experience and TPH2 genotype on HPA axis function and aggression in rhesus monkeys: A retrospective analysis. Horm. Behav. 57, 184-191 (2010).

23. Watson, K. K., Ghodasra, J. H. \& Platt, M. L. Serotonin transporter genotype modulates social reward and punishment in rhesus macaques. Plos One 4, e4156 (2009).

24. Hong, K. W. et al. Polymorphism of the tryptophan hydroxylase 2 (TPH2) gene is associated with chimpanzee neuroticism. Plos One 6, e19855 (2011).

25. Wasserman, S. \& Faust, K. Social Network Analysis: Methods and Applications (Cambridge University Press, 1994).

26. Colegrave, N. \& Ruxton, G. D. Confidence intervals are a more useful complement to nonsignificant tests than are power calculations. Behav. Ecol. 14, 446-447 (2003).

27. Cheney, D. L. Extent and limits of cooperation in animals. Proc. Natl. Acad. Sci. 108, 10902-10909 (2011).

28. Lande, R. \& Arnold, S. J. The measurement of selection on correlated characters. Evolution 37, 1210-1226 (1983).

29. Kruuk, L. E. B. \& Hadfield, J. D. How to separate genetic and environmental causes of similarity between relatives. J. Evol. Biol. 20, 1890-1903 (2007).

30. Coltman, D. W., O’Donoghue, P., Hogg, J. T. \& Festa-Bianchet, M. Selection and genetic (CO)variance in bighorn sheep. Evolution 59, 1372-1382 (2005).

31. Young, S. N. \& Leyton, M. The role of serotonin in human mood and social interaction - Insight from altered tryptophan levels. Pharmacol. Biochem. Behav. 71, 857-865 (2002).

32. Sirvio, J., Riekkinen, P., Jakala, P. \& Riekkinen, P. J. Experimental studies on the role of serotonin in cognition. Prog. Neurobiol. 43, 363-379 (1994).

33. Barr, C. S. et al. Rearing condition and rh5-HTTLPR interact to influence limbichypothalamic-pituitary-adrenal axis response to stress in infant macaques. Biol. Psych. 55, 733-738 (2004).

34. Chen, G. L. et al. TPH2 5'- and 3'-regulatory polymorphisms are differentially associated with HPA axis function and self-injurious behavior in rhesus monkeys. Genes Brain Behav. 9, 335-347 (2010).

35. Coon, H. et al. Possible association between autism and variants in the brainexpressed tryptophan hydroxylase gene (TPH2). Am. J. Med. Genet. B 135B, 42-46 (2005).

36. Canli, T., Congdon, E., Todd Constable, R. \& Lesch, K. P. Additive effects of serotonin transporter and tryptophan hydroxylase- 2 gene variation on neural correlates of affective processing. Biol. Psychol. 79 (2008).

37. Hariri, A. R. \& Holmes, A. Genetics of emotional regulation: the role of the serotonin transporter in neural function. Trends Cog. Sci. 10, 182-191 (2006).

38. Chen, G. L., Novak, M. A., Hakim, S., Xie, Z. \& Miller, G. M. Tryptophan hydroxylase-2 gene polymorphisms in rhesus monkeys: association with hypothalamic-pituitary-adrenal axis function and in vitro gene expression. Molec. Psych. 11, 914-928 (2006).

39. Jedema, H. P. et al. Cognitive impact of genetic variation of the serotonin transporter in primates is associated with differences in brain morphology rather than serotonin neurotransmission. Molec. Psych. 15, 512-522 (2010).

40. Krawczak, M. et al. Male reproductive timing in rhesus macaques is influenced by the 5HTTLPR promoter polymorphism of the serotonin transporter gene. Biol. Reprod. 72, 1109-1113 (2005).

41. Trefilov, A., Berard, J., Krawczak, M. \& Schmidtke, J. Natal dispersal in rhesus macaques is related to serotonin transporter gene promoter variation. Behav. Genet. 30, 295-301 (2000).

42. Suomi, S. J. Risk, resilience, and gene $\mathrm{x}$ environment interactions in rhesus monkeys. Ann. N.Y. Acad. Sci. 1994, 52-62 (2006).

43. Feil, R. \& Fraga, M. F. Epigenetics and the environment: emerging patterns and implications. Nat. Rev. Genet. 13, 97-109 (2012).

44. Fawcett, G. et al. Characterization of single-nucleotide variation in Indian-origin rhesus macaques (Macaca mulatta). Bmc Genomics 12, 311 (2011).
45. Rawlings, R. G. \& Kessler, M. J. The Cayo Santiago Macaques: History, Behavior \& Biology (State University of New York Press, Albany, 1986).

46. Datta, S. The acquisition of dominance among free-ranging rhesus monkey siblings. Anim. Behav. 36, 754-772 (1988)

47. UCINET for Windows: Software for Social Network Analysis (Analytic Technologies, Harvard, Massachusetts, 2002).

48. Lusseau, D., Whitehead, H. \& Gero, S. Incorporating uncertainty into the study of animal social networks. Anim. Behav. 75, 1809-1815 (2008).

49. Opshal, T. Structure and Evolution of Weighted Networks (University of London, London, 2009).

50. Mundry, R. Testing related samples with missing values: a permutation approach. Anim. Behav. 58, 1143-1153 (1999).

51. Blomquist, G. E. Fitness-related patterns of genetic variation in rhesus macaques. Genetica 135, 209-219 (2009).

52. Almasy, L. \& Blangero, J. Multipoint quantitative-trait linkage analysis. Am. J. Hum. Genet. 62, 1198-1211 (1998)

53. Rogers, J., Shelton, S. E., Shelledy, W., Garcia, R. \& Kalin, N. H. Genetic influences on behavioral inhibition and anxiety in juvenile rhesus macaques. Genes Brain Behav. 7, 463-469 (2008).

54. Amos, C. I., Elston, R. C. \& Rao, D. C. Robust methods for the detection of genetic linkage for quantitative data from pedigrees. Genet. Epidemiol. 6, 349-360 (1989).

55. Lynch, M. \& Walsh, B. Genetics and Analysis of Quantitative Traits (Sinauer Associates, 1998).

56. Widdig, A. et al. A longitudinal analysis of reproductive skew in male rhesus macaques. Proc. Roy. Soc. B. 271, 819-826 (2004).

57. Stinchcombe, J. R., Agrawal, A. F., Hohenlohe, P. A., Arnold, S. J. \& Blows, M. W Estimating nonlinear selection gradients using quadratic regression coefficients: Double or nothing ? Evolution 62, 2435-2440 (2008).

58. Rasbash, J., Steele, F., Browne, W. J. \& Goldstein, H. (Centre for Multilevel Modelling, University of Bristol, 2009).

59. Wendland, J. R. et al. Differential functional variability of serotonin transporter and monoamine oxidase a genes in macaque species displaying contrasting levels of aggression-related behavior. Behav. Genetic. 36, 163-172 (2006).

60. Lewontin, R. C. Interaction of selection and linkage 1: general considerations, heterotic models. Genetics 49, 49-67 (1964).

\section{Acknowledgments}

We thank Bonn Aure, Jacqueline Buhl, Monica Carlson, Matthew McConnell, Elizabeth Maldonado, David Paulsen, Cecilia Penedo \& the Caribbean Primate Research Center (CPRC) for assistance, and Roger Mundry for the use of PSAM software. The authors were supported by NIMH grant R01-MH089484, an Incubator Award from the Duke Institute for Brain Sciences, and a Duke Center for Interdisciplinary Decision Sciences Fellowship to LJNB. The CPRC is supported by grant 8-P40 OD012217-25 from the National Center for Research Resources (NCRR) and the Office of Research Infrastructure Programs (ORIP) of the National Institutes of Health.

\section{Authors contributions}

LJNB, SRH, JH, JHPS and MLP conceived of the study. AGR, JH and JHPS performed the serotonergic genotyping, ARL and JGM coordinated the genetic and census-based parentage assignment. All authors participated in the coordination and collection of DNA samples. LJNB designed and coordinated the collection of behavioural data, performed the social network analyses and quantitative genetic analyses, and wrote the manuscript. All authors read and approved the final manuscript.

\section{Additional information}

Supplementary information accompanies this paper at http://www.nature.com/ scientificreports

Competing financial interests: The authors declare no competing financial interests. License: This work is licensed under a Creative Commons Attribution-NonCommercial-NoDerivs 3.0 Unported License. To view a copy of this license, visit http://creativecommons.org/licenses/by-nc-nd/3.0/

How to cite this article: Brent, L.J.N. et al. Genetic origins of social networks in rhesus macaques. Sci. Rep. 3, 1042; DOI:10.1038/srep01042 (2013). 